
allemande

46-2 | 2014

Intellectuels et politique en Allemagne

\title{
Brandt und Grass - eine Freundschaft?
}

\section{Friedhelm Boll}

\section{OpenEdition}

Journals

Édition électronique

URL : https://journals.openedition.org/allemagne/1135

DOI : 10.4000/allemagne. 1135

ISSN : 2605-7913

Éditeur

Société d'études allemandes

\section{Édition imprimée}

Date de publication : 30 décembre 2014

Pagination : 347-363

ISSN : 0035-0974

\section{Référence électronique}

Friedhelm Boll, „Brandt und Grass - eine Freundschaft?", Revue d'Allemagne et des pays de lanque allemande [Online], 46-2 | 2014, Online erschienen am: 29 Juli 2019, abgerufen am 18 Mai 2021. URL: http://journals.openedition.org/allemagne/1135 ; DOI: https://doi.org/10.4000/allemagne.1135 


\section{Brandt und Grass - eine Freundschaft?}

\section{- Friedhelm Boll*}

Willy Brandt und Günter Grass bildeten eine Art Partnerschaft, wie es sie zwischen einem führenden Politiker und einem Intellektuellen in Deutschland zuvor nicht gegeben hat. Nicht weniger bedeutend ist die Quellenbasis. Neben seinen Büchern Aus dem Tagebuch einer Schnecke, in dem er den Wahlkampf von 1969 beschreibt, und seiner autobiographischen Arbeit Beim Häuten der Zwiebel hat Grass mehrfach selbst über seine politische Arbeit und seine Nähe zu Willy Brandt berichtet. Nun liegt zudem der gesamte Briefwechsel aus dem Nachlass Willy Brandts sowie aus dem Günther-Grass-Archiv vor, der eine hervorragende Basis für die Beziehungen der beiden Nobelpreisträger abgibt ${ }^{(1)}$. Vereint sind „288 Briefe, Grußkarten und Telegramme sowie Briefbeigaben und begleitende Schreiben“, die Martin Kölbel 2013 vorzüglich ediert hat. Diese Briefe sind nach Meinung des Herausgebers ein „Zeugnis einer auch gelingenden Liaison von Geist und Macht“, das „einen Einblick hinter die Kulissen der Macht“ gibt ${ }^{(2)}$. Zweifellos ist dieser von 1964 bis 1992, dem Todesjahr Willy Brandts, reichende Briefwechsel als ein wichtiges, „vielleicht sogar zentrales Dokument“ für „die zweite, die intellektuelle Gründung der Bundesrepublik“ anzusehen ${ }^{(3)}$. Das hier angeschnittene Thema umfasst daher einen zentralen Aspekt des Wandels der

* Professor an der Universität Kassel und Wissenschaftlicher Referent am Historischen Forschungszentrum der Friedrich-Ebert-Stiftung bis 2010.

1 Martin Kölbel (Hg.), Willy Brandt und Günter Grass, Der Briefwechsel, Göttingen, Steidl, 2013, S. 1059. Alle Briefe sowie die beigegebenen Dokumente von Brandt und Grass werden nach dieser Ausgabe zitiert, jeweils als Brief bzw. Dokument mit entsprechender Nummer.

2 Martin Kölbel, „Nachwort“, in: ebd., S. 1061.

3 Ebd.; zur Debatte um die intellektuelle Gründung der Bundesrepublik siehe: Klaus SchönHoven, „Aufbruch in die sozialliberale Ära. Zur Bedeutung der 60er Jahre in der Geschichte der Bundesrepublik“, Geschichte und Gesellschaft, 25. Jg., Heft 1, S. 123-145. Clemens Albrecht u.a. (Hg.), Die Intellektuelle Gründung der Bundesrepublik. Eine Wirkungsgeschichte der Frankfurter Schule, Frankfurt/ New York, Campus, 1999; Claudia Münkel, „Intellektuelle für die SPD. Die Sozialdemokratische Wählerinitiative“, in: Gangolf HüBinger, Thomas Hertfelder (Hg.), Kritik und Mandat. Intellektuelle in der deutschen Politik, Stuttgart, DVA, 2000, S. 222-239. 
politischen Kultur der Bundesrepublik zu einer partizipatorischen Demokratie, die mit der Ära Brandt untrennbar verbunden ist ${ }^{(4)}$. Die Besonderheit der Partnerschaft Brandt-Grass lässt sich mit den Worten Kölbels wie folgt beschreiben: „Ein Politiker, der die intellektuelle Dreinrede in seine Partei- und Regierungsarbeit einzubinden weiß, und ein Schriftsteller, der Gesellschaft nicht einfach künstlerisch entwerfen, sondern tagespolitisch mitgestalten will: Beides hat Seltenheitswert “(5).

Für die Analyse dieser Partnerschaft ist es sinnvoll, bei Grass mindestens vier Rollen zu unterscheiden, in denen er gegenüber Willy Brandt auftrat: Der 1999 mit dem Literaturnobelpreis ausgezeichnete Schriftsteller, der mit seinem Roman Die Blechtrommel Weltliteratur schrieb; der Intellektuelle, der öffentlich Stellung bezieht und sich einmischt; der (selbsternannte oder auch gebetene) Ratgeber des Bundeskanzlers und der Helfer bzw. Wahlkämpfer, d.h. der Erfinder und Organisator einer eigenen, historisch neuen Wahlkampfform (der Sozialdemokratischen Wählerinitiative), mit der er die SPD insbesondere zur Zeit Willy Brandts unterstützte und gleichzeitig eine Form der aktiven Mitwirkung des Wählers schafft. Dass Grass mit diesen verschiedenen Rollen gelegentlich in Konflikt geriet, wird die Darstellung zeigen. Am Schluss wird die Frage aufgeworfen, ob es sich bei dieser Partnerschaft um Freundschaft handelte, wie Grass und der Herausgeber Kölbel behaupten ${ }^{(6)}$.

\section{Einige Fakten}

Obwohl sich Grass bereits im Bundestagswahlkampf von 1961 individuell für Willy Brandt engagiert, beginnt der Briefwechsel erst 1964. Ab 1967 nimmt der schriftliche Austausch langsam zu, bevor zwischen 1969 und 1973 der Höhepunkt erreicht wird. Nach dem Rücktritt Brandts vom Amt des Bundeskanzlers 1974 reduziert sich der Briefwechsel auf wenige Kontakte und einige gemeinsame Projekte (Zusammenarbeit bei der Zeitschrift L 76), die bis ins Todesjahr Brandts (1992) reichen. Über die deutsche Einheit haben sie geradezu entgegengesetzte Ansichten.

Im Unterschied $\mathrm{zu}$ anderen kritischen Intellektuellen belässt es Grass nicht bei papiernen Stellungnahmen. Er will mehr. Es drängt ihn wie schon bei seinen Pro-SPDWahlkämpfen von 1961 und 1965 zur Politik. Nach dem Wahlsieg von 1969 möchte er unmittelbar in der Nähe des Kanzlers eine ihm angemessene politische Verwendung, die aber nie gefunden wird. Zwar bleibt immer erkennbar, dass er der SPD-kritische Kämpfer ist. Seine Aktivität als Wahlkämpfer bildet somit die zweite Rolle, die er zeitweise mit überausgroßem Engagement ausfüllt. Mit Hilfe der Sozialdemokratischen Wählerinitiative schafft er ein neues Instrument partizipativer Demokratie, mit dem er ab 1969 zu einem beträchtlichen Maß dazu beiträgt, die SPD auch für sogenannte bürgerliche Wählerschichten und für Nichtparteimitglieder zu öffnen. Von besonderer Bedeutung ist der katholische Arbeitskreis in der Sozialdemokratischen

4 K. Schönhoven, „Aufbruch“ (Anm. 3), S. 144.

5 M. Kölbel, „Nachwort“ (Anm. 2), S. 1061.

6 Ebd., S. 1060. Von Freundschaft zwischen beiden Männern, ohne dies zu problematisieren, spricht Daniela MüNKel, „,Mehr Demokratie wagen, mitarbeiten!' Günter Grass und die Sozialdemokratische Wählerinitiative“, in: Kurt BECK (Hg.), „Schlagt der Äbtissin ein Schnippchen, wählt SPD!“Günter Grass und die Sozialdemokratie, Berlin, Vorwärts-Buch Verlag, 2007, S. 41. 
Wählerinitiative, weil sich dort in herausgehobenem Maße die Kreise fanden (Theologieprofessoren, Studentenpfarrer, Vertreter katholischer Laienverbände), die sich für die Aussöhnung mit Polen einsetzten ${ }^{(7)}$.

Grass’ Drängen, politisch Einfluss zu gewinnen, führte 1967/68 dazu, sein Verhältnis zu Brandt zu intensivieren. Brandt bietet dem 14 Jahre jüngeren das Du an und akzeptiert, dass er in einem sich ständig intensivierenden Briefwechsel immer wieder konkrete Ratschläge erhält. Darunter finden sich Vorschläge, die sich langfristig als äußerst sinnvoll erweisen. Grass' Warnungen vor der Umweltzerstörung der Ostsee, seine Analyse zum Aufbegehren der Jugend gegen Ende der 1960er Jahre sowie sein Vorschlag zur Gründung einer deutschen Nationalstiftung für das deutsche Kulturgut im Osten sind besonders hervorzuheben. Hier zögert er nicht, am Beispiel seiner eigenen literarischen Produktion oder der seines Freundes Siegfried Lenz die Dimension der Erinnerung (an die verlorene Heimat) herauszustreichen, um die Bedeutung des deutschen Ostens als ein zu bewahrendes Kulturgut zu betrachten, das den ehemaligen Vertriebenen helfen kann, mit dem Verlust der Heimat besser zurecht zu kommen. Nicht selten kommen Grass' Ratschläge jedoch ungelegen. Da er die Sensibilität Brandts nicht nur kennt, sondern als dessen Stärke schätzt, muss sein forderndes Auftreten gegenüber Brandt auch bei seinem so hoch geschätzten Briefpartner nicht selten als störende Einmischung angekommen sein. Gerade im internen Verhältnis spart Grass nicht an Kritik. So stehen sinnvolle Hinweise, ungebetene Ratschläge, harte Kritik und massive Einmischung oft unverbunden nebeneinander.

Die Fälle, in denen Brandt Grass direkt als Berater anspricht, sind eher selten. Die bedeutendsten dürften die Mithilfe an der Formulierung der Warschauer Fernsehansprache vom 7. Dezember 1970, die Unterstützung bei der Formulierung der Osloer Nobelpreisrede und vielfältige andere große Reden zu Parteitagen gewesen sein. Dort wo Grass' Ratschläge im Rahmen der Wahlkampfvorbereitung und -durchführung konkret wurden, bat Brandt seine Mitarbeiter Horst Ehmke, Hans-Jürgen Wischnewski, Holger Börner oder gelegentlich auch Alfred Nau (für die Friedrich-EbertStiftung), der direkte Ansprechpartner für Grass zu sein.

Die Beziehung der beiden Männer ist seit dem Beginn in den 1960er Jahren immer wieder von Auseinandersetzungen begleitet, die in der Regel aus Grass öffentlicher Kritik an Brandts Regierungstätigkeit entstehen. Dennoch bleiben beide während der Zeit von Brandts Kanzlerschaft eng verbunden. Brandt nutzt sowohl die intelligente Analysefähigkeit, als auch die bestechenden Formulierungsvorschläge von Grass für seine großen Reden. Auch kommt dem Politiker die Unterstützung des weltbekannten Schriftstellers sowie seine Netzwerktätigkeit zu Gute. Grass macht immer wieder auch eigene Vorschläge für Reden und Stellungnahmen, die Brandt nicht selten an seine Mitarbeiter Egon Bahr, Horst Ehmke oder Hans-Jürgen Wischnewski weitergibt. Manche Briefe lässt der Bundeskanzler, der gewohnt ist zu delegieren, auch durch sein Büro beantworten. In der Regel sind Grass' von erstaunlicher Eloquenz gekennzeichneten Briefe drei bis fünfmal so lang wie die Antworten Brandts.

7 Vgl. Friedhelm BolL, „Der Bensberger Kreis und sein Polenmemorandum. Vom Zweitem Vatikanischen Konzil zur Unterstützung sozial-liberaler Entspannungspolitik“, in: DERs./Wiesław WYsOCKI/ Klaus Ziemer (Hg.), Versöhnung und Entspannung. Polnisch-deutsche Versöhnungsinitiativen der 1960er-Jahre und die Entspannungspolitik, Bonn, J.H.W. Dietz, 2009, S. 143-164. 
Die erste, zögerliche Kontaktaufnahme zwischen beiden Persönlichkeiten ging 1961 von Willy Brandt aus, der bereits zuvor über Hans-Werner Richter, den Spiritus Rector der Gruppe 47, Gesprächskontakte zu Repräsentanten des linken IntellektuellenSpektrums wie auch des Bildungsbürgertums suchte. Grass wurde auf Bitten von Brandt zum zweiten Gespräch des Kanzlerkandidaten mit Schriftstellern am 5. September 1961, also kurz vor der Bundestagswahl, eingeladen. Zu dieser Zeit nahm er auch bereits an zwei Wahlkampfauftritten von Brandt in Westdeutschland teil ${ }^{(8)}$. Die direkte Konfrontation mit Brandt und dessen Politik scheinen Grass so beeindruckt zu haben, dass er seine hochfahrende Ablehnung der Politik der 1950er Jahre verlor. In seinem Buch Grimms Wörter. Eine Liebeserklärung schreibt Grass dazu: „Brandt zeigte Verständnis für fast jede vorgebrachte Beschwerde ${ }^{\text {( }}{ }^{(9)}$. Als Brandt fragte, wer bereit sei, seine Reden durch Beiträge zu bereichern, war Grass der einzige, der sich meldete $^{(10)}$. Und er hielt Wort.

1961 fuhr er mit der Eisenbahn durchs Land, hielt Wahlveranstaltungen für die SPD und ihren Kandidaten Willy Brandt und beteiligte sich an einem von seinem Freund Martin Walser herausgegebenen Taschenbuch, in dem trotz aller Kritik für das kleinere Übel SPD geworben wurde ${ }^{(11)}$. War der Kontakt nach der Wahlniederlage Brandts zunächst abgebrochen, so weitete Grass 1965 sein Engagement aus, indem er im Kreis von Künstlern, Schriftstellern, Film- und Fernsehschaffenden für Brandt warb und eine illustre Schar von Persönlichkeiten motivierte, sich öffentlich für Brandt einzusetzen ${ }^{(12)}$.

Kennzeichnend für die erste Phase der Beziehungen zwischen Brandt und Grass ist die unterstützende Arbeit des Schriftstellers. Er hilft Brandt bei der Redaktion seiner Reden zu wichtigen Bundesparteitagen wie im Wahlkampf, wirbt für ihn in Künstlerkreisen, hält selbst-finanzierte und -verantwortete Wahlkampfreden, beteiligt sich an Taschenbüchern zur Unterstützung der SPD, konzipiert Werbeslogans für den Wahlkampf 1961, schreibt Redeentwürfe zu anderen wichtigen Themen insbesondere zum Problem der rebellischen Jugend ${ }^{(13)}$.

Brandt liefert seinerseits ein interessantes, im Vergleich zu Konrad Adenauer völlig neues Beispiel für den Kurs der Nach-Godesberg-SPD, die sich aus dem Dunstkreis der Arbeiter- und Funktionärspartei zu lösen suchte und ernsthafte Kontakte zu Wissenschaftlern, Experten sowie zu Repräsentanten der kritischen Intelligenz aufnahm. Gleichzeitig präsentiert er diese „neue“ SPD als eine Partei, die sich von der Fundamentalopposition der frühen Nachkriegszeit gelöst und mit der Betonung von

8 M. Kölbel, „Nachwort“ (Anm. 2), S. 1065.

9 Ebd., S. 1067.

10 Ebd.

11 Siehe Günter Grass, „Wer wird dieses Bändchen kaufen?“, in: Martin WALser (Hg.), Die Alternative oder Brauchen wie eine neue Regierung?, Reinbek bei Hamburg, Rowohlt, 1961. Erneut abgedruckt in: K. BЕск (Hg.), Grass und die Sozialdemokratie (Anm. 6), S. 22-24.

12 Wieder erschien ein rororo-Bändchen zur Bundestagswahl, an dem Grass beteiligt war. Vgl. HansWerner Richter, Plädoyer für eine neue Regierung oder Keine Alternative, Reinbek bei Hamburg, Rowohlt, 1965. Für den Zusammenhang: D. Münkel, „Wählerinitiative“ (Anm. 6), S. 34. Zum Pressegespräch mit Brandt u.a. erschienen: Fritz Kortner, Hans Werner Henze, Bernhard Wicki und Ingeborg Bachmann. 
deutschen und europäischen Gemeinschaftsaufgaben, einer grundlegenden Reform der Bildungspolitik und der atlantischen Partnerschaft (mit dem amerikanischen Präsidenten J.F. Kennedy) zugewendet hat. Der kritische, manches Mal auch konfliktbehaftete Dialog gerade mit Günter Grass wird ein wichtiges Merkmal seiner Kanzlerschaft bleiben.

Ein weiteres, vom Berliner Regionalsender SFB ausgestrahltes Gespräch zwischen Schriftstellern und Brandt, diesmal im Wahlkampf 1965, zeigt ein deutliches Aufeinanderzubewegen von „Geist und Macht“, für das jedoch noch ein neues Rollenverständnis zu suchen war. Dabei konnte die aus Frankreich herüberreichende Debatte um littérature engagée und littérature pure nicht weiterhelfen ${ }^{(14)}$. Brandt selbst sprach mit Blick auf die Intellektuellen von einer „Pflicht zum Engagement“ „nicht unbedingt in einer Partei, aber in der Mitverantwortung für die öffentlichen Angelegenheiten “(15). Dies kam Grass entgegen, der ein idealtypisches Rollenverständnis entwickelte, demzufolge er als Bürger politisch zu sein habe, sich als Schriftsteller jedoch an den Regeln der Kunst bzw. der Ästhetik orientieren werde ${ }^{(16)}$.

Das Verhältnis von Geist und Macht ließ sich jedoch nicht mit einem bipolaren Rollenverständnis klären. Es war zu ergründen, wie das politische Engagement von Künstlern, insbesondere von Schriftstellern aussehen könnte. Grass hat dazu keine theoretischen Ergüsse verfasst, sondern jeweils pragmatisch und direkt gehandelt. In einem langen Brief an Horst Ehmke vom 8.11.1967, damals Staatssekretär in Gustav Heinemanns Justizministerium (und mit Durchschlägen an Willy Brandt und Gustav Heinemann), fasst Grass die Diskussion zusammen, die sich zwischen Hans-Werner Richter, ihm und Ehmke entwickelt hatte. Dabei ging es um die Frage, ob Schriftsteller die großen utopischen Entwürfe zu machen haben, während Politiker für den Alltag und die Details zuständig seien. Während sich Ehmke verwundert zeigte über die Einmischung von Schriftstellern in die Tagespolitik und sie stärker auf größere „Zukunftsweisende Aufrisse, womöglich Utopien“ verweisen wollte, begrüßte Grass deren konkrete politische Stellungnahmen, auch wenn diese Einmischung nur Notlösungen seien. Dies zwinge Schriftsteller jedoch, „am täglichen Brot der Politiker mitzuknabbern und sein Gebiß am Altbackenen zu erproben“. Dennoch sei die „Detailfusselei, gemessen an zurückliegenden utopischen Fluchtversuchen, nur als das andere Extrem zu betrachten “ (17). Daher empfahl er seinen Gesprächspartnern in der SPD, den Philosophen Ernst Bloch, der soeben mit seiner Dankrede zur Verleihung des Friedenspreises des Deutschen Buchhandels (1967) Aufsehen erregt hatte, zum nächsten Parteitag einzuladen, und mit ihm „die gesellschaftsformende Kraft der SPD wieder einmal neu zu beleben“ “(18). Dazu sei es auch sinnvoll, das Godesberger Programm, von dem kaum noch jemand spreche, neu zu bedenken ${ }^{(19)}$.

14 Vgl. die Ausstellung: Dichter und Richter. Die Gruppe 47 und die deutsche Nachkriegsliteratur. Ausstellung der Akademie der Künste, Berlin, 1988, zitiert nach M. KöLbel, „Nachwort“ (Anm. 2), S. 1069.

15 Ebd., S. 1070.

16 Ebd.

17 Dokument 14: Grass an Ehmke, 8.11.1967, S. 837ff.

18 Ebd., S. 838. Die Einladung zum Parteitag erfolgte auch. Bloch unterstützte Brandt im Rahmen der Wählerinitiative öffentlich.

19 Ebd. 
Orientierte sich Grass hier noch an einem bipolaren Modell von Künstler einerseits und politisch aktivem Bürger, so trat mit der Bildung der Großen Koalition Ende 1966 eine neue Dimension hervor. In einem berühmten offenen Brief an Willy Brandt brachte er am 26.11.1966 seine sowie die Kritik anderer Schriftsteller an der erwarteten Großen Koalition wirkungsvoll zum Ausdruck. Damit trat er aus der Rolle des Helfers und Unterstützers Willy Brandts heraus und profilierte sich als wortgewaltiger Kritiker der SPD. Dieser in mehreren überregionalen Zeitungen abgedruckte Brief markiert gleichzeitig eine gewisse Abkühlung gegenüber der SPD, nicht nur von Grass. Zwei Tage nach Grass antwortete Brandt mit einem Brief, der gleichzeitig mit Grass' Schreiben am 2.12.1966 in der Wochenzeitung Die Zeit erschien ${ }^{(20)}$. Normalerweise waren Brandts Briefe äußerst kurz gehalten, hier jedoch war Brandts Brief länger als der von Grass. Inhaltlich zeigt Brandt, wie ernst er die Sorgen seines Gegenübers nahm und ebenso, wie überzeugt er vom eingeschlagenen Weg seiner Partei war.

In diesem Briefwechsel zeigt sich Grass als der große Mahner und Warner, eine Rolle, die er auch später immer wieder einnahm. Im internen Briefverkehr kommt er mehrfach auf diese Warnungen zurück, die er Brandt und auch Horst Ehmke, damals Staatssekretär, später Justizminister und unter Brandt Chef des Kanzleramts, z.T. mit gespreiztem Worten vorhält. Demgegenüber betont Brandt das, was für die Jahre von 1966-1969 wirklich entscheidend sein wird: Dass die SPD hier eine für sie entscheidende Chance erhält zu zeigen, was sie kann, ohne ihre Glaubwürdigkeit zu verlieren. Das Besondere an der Beziehungen beider Männer wird in Brandts Antwort klar: Es gelingt Brandt intern ebenso wie öffentlich, Grass mit seinen Mahnungen und Warnungen ernst zu nehmen aber gleichzeitig seine eigene Linie zu begründen und zu verteidigen. Wie die Geschichte zeigt, wird Brandt Recht behalten. Während Grass die große, teils auch theatralische Geste liebt, erreicht es Brandt zu integrieren, den internen ebenso wie den öffentlichen Dialog zu pflegen. Grass jedoch geht des Öfteren an die Grenzen ihrer Beziehung - und manchmal auch darüber hinaus.

Ein für Grass äußerst wichtiges Element seiner Argumentation ist der Hintergrund der NS-Geschichte. Brandt ist für ihn die Lichtgestalt, die das „andere Deutschland“, das Deutschland des Widerstands und des Exils repräsentiert. Er möchte dieses Bild herausgehoben und nicht durch die „Kumpanei“ mit einem ehemaligen NSDAP-Mitglied und jetzigen Kanzler beschädigt sehen. Nicht nur im literarischen Werk, auch in der Politik besitzt die Auseinandersetzung mit dem Nationalsozialismus für ihn höchste Priorität. Kurz nach Bildung der Großen Koalition fordert er Bundeskanzler Kiesinger wegen dessen NSDAP-Mitgliedschaft und seiner Arbeit für den NS-Staat als Beamter in der Propagandaabteilung des Auswärtigen Amts zum Rücktritt auf. In ähnlicher Weise reibt er sich an der NSDAP-Mitgliedschaft des nach Brandt wichtigsten SPD-Ministers Karl Schiller, dem er nahe legt, sich öffentlich zu seinem „Irrtum“ zu bekennen ${ }^{(21)}$.

20 Der Briefwechsel ist auch abgedruckt in: Pressemitteilungen und Informationen, SPD, Bonn, 28.11.1966.

21 Dokument 38, Grass an Karl Schiller, 15.7.1969, S. 892f. 


\section{Die Sozialdemokratische Wählerinitiative}

Obwohl sich Grass auch in anderen Fragen wie der turbulenten innenpolitischen Lage in West-Berlin nach dem Tod von Benno Ohnesorg (2.6.1967), der Auseinandersetzung mit der rebellierenden Jugend, dem Regime der Obristen in Griechenland und dem Vietnamkrieg (intern wie öffentlich) zu Wort meldet, gilt seine eigentliche politische Hoffnung sowie sein Engagement der Zukunft Willy Brandts sowie der SPD. Zukunftsentwürfe oder gar Visionen sind seine Sache nicht. Sein politischer Ansatz ist ein moralischer. In Brandt und der SPD sieht er das „andere“, antinationalsozialistische Deutschland, das bereit zum Frieden mit Osteuropa ist und gleichzeitig den Kampf sowohl gegen neue Diktaturen (Griechenland), verfehlte Kriegspolitik (Vietnam) und zur Unterstützung der Bürgerrechtler des Ostblocks aufnehmen sollte. Immer wieder bringt er die Gefahren der Großen Koalition zur Sprache: Radikalisierung eines Teils der Jugend nach Linksaußen, Abwanderung konservativer Kreise zur NPD, Verwässerung der sozialdemokratischen Identität. So entschließt er sich bereits im Herbst 1967 Wahlkampfplanungen für 1969 aufzunehmen: „Das Ziel kann nur heißen: Die SPD muss die Wahlen 1969 gewinnen.“ Im Rahmen der Großen Koalition müssten daher die Leistungen der SPD weit stärker hervorgehoben werden ${ }^{(22)}$. Anders als in den beiden vorangegangenen Jahren stellt er seine Aktivitäten nun auf eine breitere Basis. In Absprache mit Brandt schart er einen kleinen Kreis engagierter Schriftsteller, Wissenschaftler und Jugendvertreter um sich, die Überlegungen zur Unterstützung der SPD anstellen. In diesem Sinn arbeiten mit: als Schriftsteller außer ihm noch Siegfried Lenz (ebenso wie Grass ein Heimatvertriebener), an Hochschullehren Eberhardt Jäckel (Historiker), Kurt Sontheimer, Arnulf Baring und Heinz Josef Varain (jeweils Politikwissenschaftler), an Vertretern des Sozialdemokratischen Hochschulbundes (SHB) Erdmann Linde und Knut Nevermann sowie der Repräsentant der Parteilinken Jochen Steffen ${ }^{(23)}$. Willy Brandt bittet Horst Ehmke sowie seinen Vertrauten Leo Bauer, Chefredakteur der SPD-Zeitschrift Die Neue Gesellschaft, den Kontakt zu halten. In einer späteren Phase gelingt es, auch Herbert Wehner für die Arbeit zu gewinnen.

Als Aufgaben des Kreises wurden zunächst vorgeschlagen: a) Hilfe bei der Ausarbeitung „langfristiger politischer Vorhaben (z.B. Mitbestimmung, Studienreform usw.)“; b) verbesserte Öffentlichkeitsarbeit und c) eine bundesweite „Wahltournee von Künstlern und Professoren“, die sich insbesondere an die Zielgruppe der Neuwähler und ein linksorientiertes Bürgertum richten sollte ${ }^{(24)}$. Bereits vor diesen Tätigkeiten entstand nach einem ersten Auftritt im Landtagswahlkampf Schleswig-Holstein im Frühjahr 1967 die Idee, nicht nur eine Wahltournee mit Prominenten durchzuführen, sondern diese durch lokale Bürgerinitiativen organisieren zu lassen ${ }^{(25)}$. Das

22 Dokument 14: Brief an Ehmke, 8.11.1967, S. 838f.

23 Horst Eнмке, Mittendrin. Von der Großen Koalition zur Deutschen Einheit, Berlin, Rowohlt, 1994, S. 84 .

24 Dokument 17: Ehmke Notiz für Willy Brandt über den privaten Arbeitskreis von G. Grass, 19.2.1968, S. 846.

25 M. Kölbel, „Nachwort“ (Anm. 2), S. 1095. Kölbel zeichnet ausführlicher, als es hier möglich ist, die Entwicklung der Sozialdemokratischen Wählerinitiative nach. Vgl. auch Brief 31: Grass an Brandt, 27.4.1967, S. 149ff. 
Wort Bürgerinitiative nahm bald seiner Bedeutung entsprechend einen erweiterten Charakter an. Der private Arbeitskreis machte sich nicht nur Gedanken über einen Macht- und Politikwechsel, niedergelegt in entsprechenden Ausarbeitungen, sondern auch über den Erhalt und die Festigung der Demokratie überhaupt „mit der SPD als letzter verbliebener Retterin“(26). Die von Grass initiierten Initiativen sollten die SPD für neue Wählerschichten öffnen und damit Sympathisanten und Multiplikatoren in bisher unerreichten Wählerschichten gewinnen und diese sowohl zur SPD führen wie ihr Wirken in diese Gesellschaftsschichten hinein tragen. Grass' Leitbegriff war der des Bürgers, der die Politik nicht allein den Parteien überlassen dürfe („Politik ist Bürgersache") ${ }^{(27)}$. So gestalteten sich die im Wahlkampf 1969 gegründeten lokalen Wählerinitiativen als eine Art gesellschaftliche Erneuerung, für die Willy Brandt als Garant wirkte. Man kann sie im Nachhinein durchaus als „wichtigen Motor der zweiten, gesellschaftlichen Gründung der Bundesrepublik“ “bezeichnen ${ }^{(28)}$. Im berühmten sogenannten Willy-Wahlkampf von 1972 steigerte Grass diese Aktivität von 90 Wählerinitiativen (1969) auf 350 und schloss ihnen zusammen mit Heinrich Böll einen katholischen Arbeitskreis an ${ }^{(29)}$.

Selbstverständlich blieb Grass auch während der Zeit von Brandts Regierungstätigkeit als Schriftsteller tätig. Er hielt Lesungen ab und reiste viel ins Ausland, wo er z.B. in den USA, in Jugoslawien, der DDR, Israel und Afrika (mit in der Regel großem Erfolg) empfangen wurde und die junge westdeutsche Nachkriegsliteratur vertrat. Des Öfteren berichtete er Brandt von diesen Reisen, die er auch für politische Beobachtungen und direkte Anregungen an Brandt nutzte. Ein von Grass erstrebtes politisches Amt ließ sich nicht finden. Von Bedeutung blieb sein Werkstattbericht Aus dem Tagebuch einer Schnecke. Darin beschrieb er seinen Einsatz bei rund hundert Wahlkampfauftritten und verschränkte dies mit der literarischen Arbeit über die Verfolgung und den Abtransport der Danziger Juden. Das äußerst erfolgreiche Buch ist ein zeitgenössisch wichtiges Werk, weil in ihm der Alltag des Wahlkampfs und der politischen Arbeit vor Ort erfasst und mit Reflexionen über „Stillstand im Fortschritt“ kombiniert werden. Nicht uninteressant ist zusätzlich die Reflexion über Melancholie (am Beispiel der Dürerschen Darstellung der Melancholia), die Grass als eine Schwester der Utopie begreift ${ }^{(30)}$. Brandt, der selbst zu Depressionen neigte, schätzte diese Arbeit ${ }^{(31)}$.

\section{Grass als Sprachfinder, Anreger und Mahner}

Ab 1968, das Jahr in dem Brandt Grass das freundschaftliche Du anbietet, intensiviert sich der Briefwechsel. Es kommt (noch) nicht zu Konflikten wie ab 1971, wohl aber zu produktiver Kooperation. Grundsätzlich enthalten die oft vier bis sechs Seiten

26 So M. KöLbel, ebd., S. 1099. Der engere Kreis schrieb auch eine ganze Reihe von z.T. sehr SPD-kritischen Positionspapieren.

27 Ebd., S. 1101.

28 So M. Kölbel, ebd., S. 1105.

29 Für die Zahlen siehe: D. MüNkel, Wählerinitiative (Anm. 6), S. 48.

30 Vgl. M. Kölbel, „Nachwort“ (Anm. 2), S. 1127ff. Sowie die Briefe 131 und 219, jeweils Brandt an Grass.

31 Ebd., S. 1128. 
langen Grass-Briefe ein Spektrum an Kritik, Vorschlägen und sprachlich glänzenden Formulierungen. Selten findet sich ein Brief, der nicht auf einen Grundton von Kritik gestimmt ist - Kritik, die immer als Anregung zur Verbesserung der Partei- bzw. Regierungsarbeit von Brandt gedacht ist. Der Herausgeber des Briefwechsels sieht die eigentliche Stärke von Grass in „der Sprache des Politischen“. In dieser Formulierungsgabe sei Grass zwar „in Brandts Politik unscheinbar“ aber „am präsentesten“ gewesen ${ }^{(32)}$. Die Briefedition enthält vermutlich alle auffindbaren Entwürfe und Texte (meist in direkter Gegenüberstellung), die Grass Brandt zu bestimmten Anlässen hat zukommen lassen. Aus Platzgründen kann hier nur eine kleine Auswahl geboten werden. Diese Vorlagen beziehen sich auf Wahlkämpfe, große Parteitagsreden und wichtige öffentlichen Reden, wie z.B. die Warschauer Fernsehrede vom Dezember 1970, mit der Brandt von Warschau aus den Abschluss des Vertrags und die Anerkennung der Oder-Neiße-Grenze rechtfertigt, oder auch die Nobelpreisrede 1971. Von Gewicht sind die Formulierungen, die Grass findet, um den Kniefall Brandts vor dem Denkmal der Ghettokämpfer in Warschau zu beschreiben. Brandt betont, der Kniefall sei nicht geplant gewesen. Somit mögen ihm auch die Worte gefehlt haben, ihn zu erklären. Für eine später zu haltende Rede schlägt Grass folgende Formulierung vor: „Als ich Anfang Dezember in Warschau stand, lag diese Last auch auf mir. Ich habe getan, was Menschen tun, wenn die Worte versagen: ich habe das Knie gebeugt und der Millionen Ermordeter gedacht“( ${ }^{\text {(33) }}$. Dass Brandt diese Formulierung in veränderter Form übernimmt, zeigt, wie sehr ihm Grass aus der Seele gesprochen hatte. In seinen Erinnerungen betont Brandt, er habe die Besonderheit des Gedenkens am Ghetto-Denkmal zum Ausdruck bringen müssen. Dann fährt er fort: „Am Abgrund der deutschen Geschichte und unter der Last der Millionen Ermordeter tat ich, was Menschen tun,

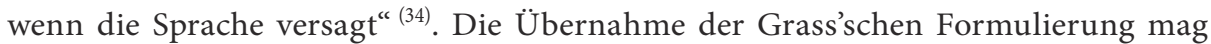
darauf hindeuten, dass Brandt zunächst nicht recht wusste, wie er seine sprachlose Geste beschreiben sollte.

Grass' Briefe sind nicht nur auf Kritik gestimmt ${ }^{(35)}$, sie enthalten neben konkreten Anregungen auch z.T. äußerst kritische Mahnungen und verletzende Bemerkungen. Der Schriftsteller begreift sich sowohl als Helfer (bei großen Reden) und Unterstützer, als auch als Mahner und Kritiker, der unaufgefordert Vorschläge macht, sich aufdrängt, sich schon mal im Ton vergreift, Belehrungen abgibt, Noten verteilt und wenn es nicht anders geht, auch mit einem Interview, einem Artikel oder einer Presseerklärung an die Öffentlichkeit tritt. Man kann sich gut vorstellen, dass er in der Umgebung Brandts als Nervensäge wahrgenommen wird. Diese Art des Vorgehens und der anmaßende Ton entwickelten sich seit seiner offenen Kritik an der Großen Koalition. Als ein Beispiel von vielen sei ein Brief an Ehmke vom 8.11.1967 erwähnt. Er betont die Selbstverständlichkeit, dass die SPD die Wahlen von 1969 nur gewinnen könne, wenn die Leistungen der SPD in der Koalition herausgehoben werden: „Das Erstaunen über Willy Brandts Leistungen als Außenminister (der kann das ja) beweist, wie

$32 \quad E b d .$, S. 1119.

33 Ebd., S. 1122. Hervorhebung F.B.

34 Willy Brandt, Erinnerungen, Berlin/Frankfurt am Main, Propyläen, 1989, S. 214.

35 M. Kölbel, „Nachwort“ (Anm. 2), S. 1125. 
wirksam die jahrelange Diffamierung gewesen ist ${ }^{\text {“(36) }}$. Leider spiegele sich die Arbeit Käte Strobels überhaupt nicht in der Öffentlichkeit. Zweimal betont Grass, mit seinen Warnungen vor der Großen Koalition Recht behalten zu haben. Es dominiere das „Semi-Politische“, das „Verwaschene“, und außerdem greife die von ihm prognostizierte Zerstrittenheit der CDU/CSU auf die SPD über: „gehässiges Gegeneinanderarbeiten“ bei Helmut Schmidt und K. Schiller, „eruptive Ausbrüche“ H. Wehners, die Klaus Schütz in Berlin das Leben schwer machten, „höfisches Gezänk“ zwischen Partei und Gewerkschaften und Eingriffe von oben nach unten durch Willy Brandt bei der Nominierung von Helmut Schmidt zu seinem Stellvertreter zählt er als Minuspunkte auf ${ }^{(37)}$.

Das Anmaßende seines Auftretens zeigt sich auch in einem Brief vom 9.12.1969. Kurz nach Übernahme der Kanzlerschaft startete Brandt mit wichtigen Initiativen in die ersten hundert Tage seiner Arbeit (Beginn der Neuen Ostpolitik, Innere Reformen, Rede vor dem Europarat, Treffen mit den Präsidenten der EWG-Staaten zur Erweiterung der europäischen Gemeinschaft um Großbritannien und um skandinavische Länder). Wenige Tage nach dem berühmten Treffen mit Präsident Pompidou am 1.-2.12.1969, bei dem die Erweiterung der EWG beschlossen wurde, beschwerte sich Grass über ein dreimaliges Verschieben der Begegnung mit Brandt wegen dringender Regierungsgeschäfte. „Diese Arbeitsweise“ sei „auf Dauer gesehen, ziemlich unproduktiv“ (38). Daher drängte Grass auf regelmäßige Treffen, die dann auch gelegentlich stattfanden. Grass behält auch in den folgenden Jahren die oben beschriebene Linie bei. Dies führt schon im ersten Jahr der Brandt-Regierung zu einem heftigen Konflikt mit Horst Ehmke, dem wichtigsten Mitarbeiter Brandts im Kanzleramt. Der bereits seit 1968 schwelende Konflikt eskaliert im Oktober 1971, als Grass anlässlich einer Buchvorstellung in Paris der Zeitschrift L'Express ein Interview gibt. Auf die Frage, ob er auch etwas Kritisches sagen könne, spricht er die angebliche Schwäche Brandts an, seine Ministermannschaft zu wenig zu führen und Konflikte zwischen den Ministern nicht zu unterbinden. Eine sozialdemokratische Regierung dürfe sich „den Luxus innerer Streitigkeiten wie am Habsburger Hof “ nicht leisten ${ }^{(39)}$.

Besonders hart ging Ehmke in persönlichen Gesprächen mit Grass ins Gericht. In einem Brief an Brandt berichtet Grass darüber. „Er [Ehmke] ist der Meinung, es sei sinnlos und natürlich schädlich, Dir solche Briefe zu schreiben“ (40). In einer langen Rechtfertigung spielt Grass das Express-Interview herunter, er habe dort lediglich den Missbrauch von Brandts Toleranz durch andere Minister kritisiert ${ }^{(41)}$. Im Interview hatte er jedoch wörtlich gesagt: „Willy Brandt ist ganz und gar verantwortlich für diesen Zustand“, weil er nicht hart durchgreife und den Prestigestreitigkeiten seiner Minister

36 Dokument 14, S. 839. Hier auch alle folgenden Zitate aus diesem Brief.

Ebd. In einem Brief an Ehmke vom 28.9.1969 mischt er sich aktiv in die Regierungsbildung ein, geißelt „lächerliche Machtkämpfe“ bei den verschiedenen Regierungsmitgliedern, warnt vor Conrad Ahlers Opportunismus und spricht unumwunden von „Willy Achillesferse“: der „Personalpolitik“. 
kein Ende setze ${ }^{(42)}$. Außerdem rechtfertigt sich Grass mit dem Hinweise, er sehe sich als schlechter Freund, „wenn ich Dir gegenüber in meinen Briefen, aber auch in der Öffentlichkeit meine, wie ich meine, begründete Kritik unterlassen wollte“. - Danach folgt eine Passage, die das starke Selbstbewusstsein offenbart und das grundlegend für sein gesamtes Wirken im Umfeld von Brandt anzusehen ist. Grass schreibt: „Kurzum: Ich habe es immer (und gestützt auf Deine Reaktionen und Gegenfragen) für nützlich gehalten, Dir offen und ungeschminkt zu schreiben, das heißt, Dir Bericht zu geben, wie sich sozialdemokratische Politik unter Deiner Führung im Land auswirkt" (43). Genau diese Selbsteinschätzung, besser als andere zu wissen, wie Brandts Politik beim Wähler ankomme, war es, die Brandts Mitarbeiter erregte.

Tatsache ist, dass Brandt Grass in keiner Weise kritisierte, noch ihn gar zurückwies oder den Kontakt abbrach. Zwei Tage später antwortete er, es gebe keinen Grund für Missverständnisse. „Selbstverständlich musst Du sagen und schreiben können, was Du für richtig hältst." Freimütige und freundschaftliche Kritik deprimiere ihn nicht, er frage sich nur manchmal, „wie wir zu einem besseren Informationsstand unter den Beteiligten kommen und wie wir die vorhandenen Kräfte möglichst wirksam werden lassen können“. Brandts Kritik wurde auf ein Kommunikationsproblem reduziert, dem noch die Bitte an Grass folgte, wieder einen „unbeschwerten Gesprächs- und Arbeitskontakt“ zu Ehmke herzustellen ${ }^{(44)}$. Seine Einschätzung des Konflikts zwischen Ehmke und Grass ist in eine vorsichtige Bemerkung verpackt, so dass Grass sie möglicherweise überhört hat.

Der Höhepunkt der öffentlichen Kritik an Brandt beweist im Nachhinein, dass Brandt sich trotz seiner lobenden Worte für Grass doch wohl sehr getroffen gefühlt haben muss. Nach dem fulminanten Wahlsieg von Herbst 1972, wo Brandt mit 45,8\% das höchste Wahlergebnis einfährt, das die SPD je in ihrer Geschichte erhielt, nimmt die Zusammenarbeit mit Brandt ab und die Grass'sche Kritik zu ${ }^{(45)}$. Als Beispiel sei ein kritischer Kommentar erwähnt, den Grass auf Anfrage von Peter Merseburger in der Sendung Panorama am 26.11.1973, ein Jahr nach der Bildung der zweiten Regierung Brandt/Scheel, von sich gab. Die Erklärung wurde von Grass persönlich in seinem Atelier verlesen und dadurch mit der Aura einer "großen moralischen Sendung“(46) versehen. Unter dem Titel „Koalition im Schlafmützentrott“, erschien sie kurz darauf im Vorwärts; was Brandt enorm erboste, wie Klaus Harpprecht notiert ${ }^{(47)}$. Grass tischte alle die Kritikpunkte auf, die er jahrelang Brandt mitgeteilt und z.T. auch öffentlich gemacht hatte ${ }^{(48)}$. Die Zurechtweisungen, die er von Ehmke und anderen erfahren

42 Dokument 63, S. 944.

43 Brief 147 (Anm. 40), S. 505.

44 Brief 149: Brandt an Grass, 15.10.1971, S. 508.

45 M. Kölbel spricht von einer Veränderung der „politischen Präferenz“ bei Grass „weg von der Parteinahme hin zur Regierungskritik“, die zunehmend auch eine Brandtkritik war. Vgl. M. KöLBEL, „Nachwort“ (Anm. 2), S. 1133.

46 Ebd., S. 1134.

47 Klaus Harpprecht, Im Kanzleramt. Tagebuch der Jahre mit Willy Brandt, o.O. Berlin, Rowohlt, 2000, S. 452 .

48 Das Dokument ist komplett abgedruckt bei Arnulf BARIng, Machtwechsel. Die Ära Brandt-Scheel, Stuttgart, DVA, 1982, S. 631-633. Dort auch alle folgenden Zitate. 
hatte, scheinen wieder auf: Brandt lasse sich „Abschirmung durch übereifrige Berater gefallen“ (49). Er strahle nicht „Tatkraft“ sondern „Lustlosigkeit“ aus. Die eigentliche Domäne des Kanzlers, die Entspannungs- und Europapolitik werde nach „geradezu Metternichschen Richtlinien“ ausgelegt ${ }^{(50)}$. Zum Schluss greift Grass nochmals Brandt persönlich in dem Bereich an, der sein Markenzeichen war, dem Ausgleich zwischen Moral und Macht. Wenn in Griechenland NATO-Panzer gegen Studenten eingesetzt werden, so sei „es Pflicht des Bundeskanzlers und seines Außenministers, vernehmbar Einspruch zu erheben“ (51). Beide Ämter zeichneten sich jedoch durch „beklommene Sprachlosigkeit und ängstliches Wegducken“ aus. Ganz im Stil des enttäuschten Anhängers formuliert dann Grass abschließend: „Wer Willy Brandt freundlich gesonnen ist, wird ihn an Ansprüche erinnern, die er selbst gesetzt hat.“

Brandt ist sehr betroffen, äußerte dies jedoch nicht. In der handschriftlichen Liste Brandts zu den Ereignissen, die zu seinem Rücktritt geführt haben, taucht aber auch Grass auf. Neben den Pannen und Ungeschicklichkeiten des Verfassungsschutzes, für die letztlich Hans-Dietrich Genscher verantwortlich war, und dem Treubruch eines Herbert Wehner reiht er diejenigen ein, die sich an Herbert Wehner anhängten, „ohne immer zu wissen, was sie taten“. Neben Presseorganen wie Der Spiegel, Stern erwähnt Brandt u.a. „Günter Grass: ,Denkmal“ + andere Klugscheißereien“ (52). Im Rückblick sieht Brandt somit auch Grass als einer derjenigen, die ihn zermürbt und zu seinem Rücktritt beigetragen haben ${ }^{(53)}$.

Hat Brandt Grass gegenüber diese Einschätzung je zum Ausdruck gebracht? Offensichtlich nicht, denn Brandt hat wenige Tage nach dem Auftritt bei Panorama bei einem Abendessen zu Grass einen Satz gesagt, den man nur als ironisch bezeichnen kann: „Günter es ist immer wieder gut zu wissen, daß man sich in schwierigen Lagen auf seine Freunde verlassen kann“ “(54). Von einer Tageszeitung auf die Grass-Kritik angesprochen, meinte Brandt: „Wenn ich den Vergleich einmal so ziehen darf: Ich bin besser in der Politik als im Romaneschreiben“ ${ }^{(55)}$. Über ein zu dieser Zeit stattgefundenes persönliches Treffen ist nichts veröffentlicht worden. Grass hat später erklärt, ein Mitarbeiter Brandts habe ihn zur Rede gestellt und gemeint, wie er die SPD in der Öffentlichkeit so angreifen könne, er sei dem Kanzler in den Rücken gefallen. Grass dürfte die Schelte

49 Selbst die ausgefallenen Gesprächstermine seien erwähnt worden, notiert M. KöLBEL, „Nachwort“ (Anm. 2), S. 1134. Dieser Aspekt fehlt jedoch in dem bei Baring abgedruckten Vorwärts-Artikel.

50 Zum Vorgang vgl. ebd., S. 1129ff.

51 A. BARING, Machtwechsel (Anm. 48), S. 633.

52 Mit „Denkmal“ war Grass Äußerungen gemeint, der Kanzler sei „entrückt“. Das gesamte Dokument aus handschriftlichen Notizen Brandts ist abgedruckt bei: Willy BRANDT, Mehr Demokratie wagen. Innen und Gesellschaftspolitik 1966-1974, bearb. von Wolther von KieseritzKy, Bonn, J.H.W. Dietz (Berliner Ausgabe, Bd. 7), 2001, S. 508-537, hier: S. 530.

53 Seitdem hat sich bei Brandt-Kennern der Begriff Klugscheißer für Grass breit gemacht. Vgl. Klaus HarpPrecht, Die Zeit, 27.12.2001, S. 46, der schreibt: „Kopfschüttelnd begegnen wir der grämlichen ,Klugscheißerei“ des ewig beleidigten Günter Grass, die ungeahnten Schaden anrichtete [...].“ Michael JüRGS, Bürger Grass. Biographie eines deutschen Dichters, München, Bertelsmann, 2002, S. 226, datiert das Wort noch früher. Schon vor seinem Rücktritt soll Brandt gesagt haben, „man möge ihm jetzt endlich diesen Klugscheißer vom Leib halten“. 
des Mitarbeiters nicht ernst genommen haben, denn er fügt hinzu: „Der Mann hieß Guillaume“ “(56). Damit wollte er wohl sagen: Den Mann ist ohnehin ein Verräter.

Das Brandt'sche Wort von den Klugscheißereien, das erst nach seinem Tod veröffentlicht wurde, umschreibt wohl genau das, was viele Personen im Umfeld von Brandt schon seit längerem glaubten: Dass Grass als Kritiker Brandts sich in vielen seiner Urteile nicht auf große, die Republik beschäftigende Themen konzentrierte, wie bei seinen Warnungen vor der Großen Koalition, sondern zunehmend auf die in der Meinungspresse hochgespielten Klischees verfällt ${ }^{(57)}$ oder Themen aufgreift, deren Zusammenhänge ihm nicht oder nur aus der Presse bekannt sind. Gerade die Kritik an der vorsichtigen Griechenlandpolitik von Brandts Regierung scheint Grass nicht klar gewesen zu sein. Im März 1972 hielt Grass in Athen eine Rede vor einer regimekritischen Gesellschaft. Nach Angriffen von Grass auf die Militärregierung und an die Adresse der USA wurde diese Gesellschaft verboten und sieben Mitglieder, darunter bekannte Sozialdemokraten, verhaftet. Brandt hatte daraufhin Grass in einem persönlichen Gespräch erläutert, dass er skeptisch sei gegenüber dieser Art von „demokratischem Interventionalismus “(58). - Es wäre für die Bundesregierung damals durchaus kontraproduktiv gewesen, neben schwierigen und von der amerikanischen Administration kritisch betrachteten Ostpolitik eine weitere Konfliktfront mit den USA aufzumachen.

Brandts Hemmungen, dem Kritiker Grass mit klaren und offenen Worten zu begegnen, ist schwer zu verstehen. Arnulf Baring beantwortet diese Frage mit der Bemerkung, dass Brandt in einer ihm persönlich gefährdenden Maße tolerant war ${ }^{(59)}$. Er habe alle Kritik, die man ihm entgegenbrachte, widerspruchslos hingenommen. Baring bezieht sich mit seiner Bemerkung auf Helmut Schmidt, der Brandt wegen dessen mangelnder Durchsetzungskraft kritisierte ${ }^{(60)}$. Vielleicht hat Brandt die Arbeitsteilung, wie sie sich im Dialog der offenen Briefe von 1967 eingestellt hatte, als Modell des Zusammenwirkens von Geist und Macht verstanden. So hat er des Öfteren Grass wegen seiner kritischen Äußerungen mit dem Hinweis auf dessen schriftstellerische Unabhängigkeit verteidigt. Aber 1973 hatte sich die Gesamtlage der Brandt'schen Regierung völlig verändert. Grass bleibt seiner Rolle als Berufskritiker so sehr verhaftet, dass er nicht erkennt, wie sehr Brandt nicht der Kritik, sondern der Hilfe bedarf ${ }^{(61)}$. Im Gegenteil, er spielt seine Rolle unbeirrt weiter, fällt Brandts Mitarbeitern auf die Nerven und verletzt Brandt aufs Tiefste. So trägt er dazu bei, dass der resignierende Brandt sich nicht mehr wehrt und ein halbes Jahr später seinen Abschied nimmt ${ }^{(62)}$.

56 Ebd., S. 1136 mit Bezug auf eine Äußerung von Grass in der Zeitschrift Der Spiegel.

57 Vgl. die entsprechenden Artikel des Spiegel, zitiert bei ebd., S. 1134ff.

58 Dokument 71: Brandt Vermerk über ein Gespräch mit Günter Grass, 26.3.1972, S. 956f. Vgl. die Darstellung von M. KöLBEL, „Nachwort“ (Anm. 2), S. 1132f. mit Auswertung der Kritik des Auswärtigen Amts am Verhalten von Grass.

59 A. BARING, Machtwechsel (Anm. 48), S. 633.

60 Ebd.

61 Allgemein zu Intellektuellen als Kritiker: M. Rainer LePsıus, „Kritik als Beruf. Zur Soziologie der Intellektuellen“, Kölner Zeitschrift für Soziologie und Sozialpsychologie, Heft 1, 1964, S. 75-91.

62 Zu den Faktoren des Rücktritts vgl. Peter Merseburger, Willy Brandt 1913-1992. Visionär und Realist, Stuttgart/München, DVA, 2002, S. 633, 712. Dort wird auch der Angriff von Grass erwähnt. 


\section{Schlussbetrachtung}

Offenbar hat Grass seine Kritiker-Rolle nicht hinreichend reflektiert. Zwar konnte Brandt die Diskrepanz zwischen Geist und Macht verringern, aufheben konnte er sie nicht. Einer europäischen Mittelmacht, die darum kämpfen musste, ihre eigenen Interesse im Ost-West-Konflikt zu artikulieren und - nicht zuletzt unter Aufgabe ihrer Lebenslügen wie der Hallstein-Doktrin und der Nichtanerkennung der Oder-NeißeGrenze - offensiv zu vertreten, konnte sich nicht zusätzliche zur führenden Kritikerin der USA (Vietnam, Griechenland) aufschwingen. So lässt sich seine Brandt-Kritik auch lesen als Abrechnung mit einem Politiker-Ideal, an dem er selbst mitgearbeitet hatte und das er nun aus Enttäuschung fallen ließ bzw. auf die von ihm erwartete Ideal-Linie zurück beordern wollte.

Warum sich Grass so engagiert für Brandt eingesetzt hat, ist nicht leicht zu ergründen. Zunächst dürfte Grass frühe Grundentscheidung, die SPD als seine politische Heimat zu betrachten, eine zentrale Rolle gespielt haben. Nach den Erfahrungen der beiden deutschen Diktaturen, so unterschiedlich sie auch waren, schien Grass die SPD die einzige Partei gewesen zu sein, die gleichzeitig Freiheit und soziale Gerechtigkeit glaubwürdig vertreten konnte. In der Auseinandersetzung mit den extremen Linken in Berlin, verteidigt er sein Festhalten an der SPD äußerst wortreich.

Als zweites scheint er in Brandt eine moralische und intellektuelle Größe gesehen zu haben, die ihn überzeugte. Brandt hatte, wie Grass es einmal formulierte, „die seltene Gabe, Zukunft näher heranzurücken, schemenhafte Hoffnungen und Gefährdungen zu konturieren“ (63). In diesem Sinne gelang es Brandt, die Kluft zwischen Geist und Macht zu verringern. Er band Intellektuelle, Dichter und Künstler auf eigenartige Weise an sich ${ }^{(64)}$. Dies gelang ihm besonders bei dem zur politischen Macht drängenden Grass, der ihm in dieser Hinsicht wiederum eine außerordentliche Stütze war. Zweifellos war Grass äußerst angetan von der Tatsache, der politischen Macht so nah wie möglich zu sein. Es schmeichelte ihm, Brandt nahezustehen, genauso wie es Brandt schmeichelte, gute Kontakte zu den Vertretern der kritischen Intelligenz zu haben ${ }^{(65)}$. In diesem Feld war Grass eindeutig der für Brandt wichtigste Mittler, viele Kontakte z.B. zu Golo Mann und Luise Rinser kamen jedoch auch ohne Grass zustande ${ }^{(66)}$.

Es gab aber noch ein Viertes, was Grass offenbar faszinierte: Brandt hatte sich als Gegner Hitlers bewährt, ja er repräsentierte auf besonders überzeugende Weise das, was Grass nicht war, er war überzeugter Antifaschist. Die Erblast der NS-Vergangenheit wirkt bei Grass so stark nach, dass er 1989 eine völlig andere Auffassung zur deutschen Einheit entwickelt als Brandt: Während Brandt für die rasche Einheit plädiert („Jetzt wächst zusammen, was zusammen gehört“), erhob Grass wegen der Last der deutschen Vergangenheit lautstark seine Stimme für den Fortbestand zweier deutscher Staaten ${ }^{(67)}$.

63 So P. Merseburger, Brandt (Anm. 62), S. 8.

64 Ebd.

65 Brief 153: Brandt an Grass, 18.11.1971, S. 518f.

66 Brief 103: Brandt an Grass, 17.7.1970, S. 385; Brief 143, Brandt an Grass, 28.9.1971, S. 489. Golo Mann hatte bereits seit 1969 gelegentlich bei der Sozialdemokratischen Wählerinitiative mitgearbeitet.

67 Dazu ausführlich M. Kölbel, „Nachwort“ (Anm. 2), S. 1139-1144. Offenbar brannte in Grass' Seele eine Art Feuer aus Scham und Schuld, weil er sich als Siebenzehnjähriger auf die Nazis eingelassen 
Aber wie sah es nun aus mit der Freundschaft dieser beiden in ihrer Art so verschiedenen Männern? Vom Ende der Partnerschaft her betrachtet fällt es schwer, von Freundschaft zu sprechen. Grass benutzte den Terminus nicht selten ${ }^{(68)}$, Brandt nur in wenigen Ausnahmefällen z.B. in einem Geburtstagstelegramm von 1971: „LIEBER GUENTHER ZU DEINEM 44. GEBURTSTAG MEINE HERZLICHE GRATULATION UND MEINEN AUFRICHTIGEN DANK FUER ZUSAMMENARBEIT UND FREUNDSCHAFT DU SOLLST GERADE WISSEN WIE WICHTIG ES MIR IST DASS WIR ES UNS NICHT ZU LEICHT MACHEN “ (69). So fasste er die spannungsreiche Partnerschaft ihrer beider Beziehungen. Hier wie in anderen Situationen lag die Kunst des Politikers Brandt darin, Grass persönlich - und auch als Kritiker - einzubinden und auf politische Zielsetzung zu hin zu orientieren. Ganz offenbar hat Brandt die Rolle des Kritikers, die Grass neben seinen anderen Rollen spielte, grundsätzlich akzeptiert, und vermutlich bis 1972 auch gut geheißen. Insgesamt wird man dem Herausgeber des Briefwechsels zustimmen müssen, dass das, was beide unter ihrer Freundschaft verstanden, „schwerlich auszumachen“ ist ${ }^{(70)}$. Denn Brandt hielt Grass auch auf Distanz ${ }^{(71)}$.

Die krisenhafte Zuspitzung der Partnerschaft im Jahre 1973 zeigt, dass Grass mit den verschiedenen, von ihm eingenommenen Rollen in Konflikt geriet. Hätte Brandt von einem Freund nicht erwarten können, dass er schweigt, wo seine Regierungstätigkeit ernsthaft bedroht war? Aber diese Dimension von Freundschaft war in Grass' Rollenkonzept nicht vorgesehen.

Im Prozess der Selbstfindung und der intellektuellen Neugründung der Bundesrepublik der 1960er und 1970er Jahre spielte Grass wegen seines Talents, seines Drängens in die politische Aktivität und seiner Nähe zu Brandt eine zentrale Rolle. Aus dem Kreis der Gruppe 47 war er es, der nicht als erster aber dafür mit größtem Engagement für die politische Alternative der SPD und ihres Vorsitzenden eintrat. Damit hat er „die intellektuelle Selbstanerkennung der Bundesrepublik als westliche Demokratie“ (Klaus Schönhoven) in starkem Maße beeinflusst. Auch für die politische Entwicklung eines beträchtlichen Teils der studentischen Jugend zur partizipatorischen Demokratie hat sein Wirken an der Seite Brandts ebenso wie sein Engagement in der Sozialdemokratischen Wählerinitiative äußerst wichtige Impulse gegeben. Die Neuorientierungen in der „Zweiten formativen Phase“ der Bundesrepublik werden sowohl mit dem Namen Brandt wie mit dem von Grass verbunden bleiben.

Natürlich lässt sich auf Grund des Gesagten die Frage aufwerfen, ob Brandt überhaupt zur Freundschaft mit Grass fähig war. Ganz ohne Zweifel hat ihm die Unterstützung durch Grass geholfen. In manchen Zeiten mag Grass sehr stark zum positiven Image Brandts als dem Politiker, der Geist und Macht zu versöhnen scheint und die

und Mitglied der Waffen-SS geworden war. Vgl. dazu die Autobiografie von Günter Gr Ass, Beim Häuten der Zwiebel, Göttingen, Steidl, 2006.

68 M. Kölbel, „Nachwort“ (Anm. 2), S. 1124.

69 Brief 182: Brandt an Grass, 16.10.1971, S. 509.

70 M. Kölbel, „Nachwort“ (Anm. 2), S. 1127. Der Grass-Biograph kommt auf die Frage nach der Freundschaft beider zu folgendem Urteil: „Falls Brandt überhaupt zu einer Freundschaft fähig war, was alle bezweifeln, die ihn gut kannten wie sein Freund Egon Bahr, dann lautet die Antwort Ja, kann sein, ist auch egal.“ M. Jürgs, Bürger Grass (Anm. 53), S. 224.

71 Siehe M. Kölbel, „Nachwort“(Anm. 2), S. 1127. 
moralische Dimension in der Politik repräsentiert, enorm beigetragen haben. Grass hat vom Kontakt zu Brandt keineswegs weniger profitiert, hat er doch sein Image als einflussreichen Schriftsteller gerne gesehen und hervorgehoben.

Das Geheimnis der Beziehung zwischen Brandt und Grass scheint wohl in den Momenten gelegen zu haben, in denen beide miteinander kooperierten und Gedanken zu formulieren suchten, die jenseits politischer Rituale und Sprechweisen den Bürger unmittelbar erreichten. Brandt hat das Grass'sche Sprachvermögen einmal so formuliert: „Aber er entdeckte und formte ein Talent, dessen Erfüllung Grass wohl selbst als eine Art Glück erkannt haben muss: Die Gabe, das Wort unmittelbar in Wirkung umzusetzen“(72).

\section{Zusammenfassung}

Die beiden Nobelpreisträger Willy Brandt und Günter Grass bildeten zwischen 1965 und 1974 eine Art Partnerschaft, wie es sie zwischen einem führenden Politiker und einem Intellektuellen in Deutschland zuvor und auch danach nicht gegeben hat. Viele Beobachter sprechen im Anschluss an Grass von Freundschaft. Der Autor untersucht die Partnerschaft genauer und stellt fest, dass man nur eingeschränkt von Freundschaft sprechen kann. Dabei werden vier Rollen herausgearbeitet, in denen Grass Brandt gegenübertrat: Grass war der weltbekannte Schriftsteller, der kritische Intellektuelle, der öffentlich Stellung bezieht und sich einmischt, der selbsternannte oder gebetene Ratgeber und der Unterstützer und Wahlkämpfer, der sich von 1961 bis 1972 persönlich für Brandt einsetzte. Beide Persönlichkeiten haben von dieser Partnerschaft enorm profitiert, Brandt durch den Wahlkämpfer und den z.T. grandiosen Schriftsteller, der bei vielen seiner großen Reden wichtige Formulierungen geliefert hat. Die Rolle des "Sprachfinders“ dürfte die ertragreichste Funktion von Grass gewesen sein. Aber auch der Schriftsteller hat von der Nähe zu Brandt an Renommee und Einfluss gewonnen. Da er ab 1973 jedoch immer stärker seine Funktion als Kritiker herauskehrte, kam die Beziehung in eine schwelende Krise. Brandt nannte Grass' harsche Kritik „Klugscheißereien“, wobei u.a. Letztere zu seinem Amtsverzicht 1974 führten. - Die Partnerschaft zwischen beiden Männern hat dennoch stark zur „intellektuellen Selbstfindung der Bundesrepublik als westliche Demokratie" (Klaus Schönhoven) in den 1960er Jahren beigetragen.

\section{Résumé}

Willy Brandt et Günter Grass formèrent de 1965 à 1974 un partenariat entre homme politique et intellectuel absolument inédit en Allemagne. De nombreux observateurs, à la suite de Grass, parlent d'amitié. Une analyse approfondie montre cependant que les relations entre les deux personnages étaient en réalité bien plus complexes. L'auteur analyse les différents rôles de Grass: l'écrivain reconnu internationalement, qui augmenta la renommée de l'homme politique, l'intellectuel, toujours prêt à critiquer l'homme politique, le conseiller et enfin l'allié lors des campagnes électorales. Tous deux profitèrent de cette relation étroite. Brandt bénéficia de l'aide et du talent de l'écrivain, qui fut à l'origine de nombreuses formules clé de ses grands discours. Ensemble, les deux 
hommes élaborèrent un langage politique nouveau et parfois couronné de succès. Grass tira lui aussi avantage de cette relation extraordinaire. Lorsqu'à partir de 1973, il s'affirma de plus en plus dans son rôle d'intellectuel critique, le partenariat connut une phase critique. Grass, auteur d'articles au contenu extrêmement virulent, fut surnommé par Brandt "Monsieur Je-sais-tout " ("Klugscheißer »). Ses critiques contribuèrent sans doute à ce que Brandt démissionne. Malgré cet épilogue, le partenariat extraordinaire entre ces deux personnages participa au processus intellectuel à l'issue duquel la RFA devint à ses propres yeux une "démocratie de l'Ouest" (Klaus Schönhoven).

\begin{abstract}
From 1965 to 1974 Willy Brandt and Günter Grass formed a partnership between a politician and an intellectual previously unheard of in Germany. Numerous observers, after Grass, qualified this partnership of friendship. A more thorough analysis shows however that the relationships between the two men were in reality much more complex. The author analyses the different sides to Grass: the internationally recognized writer, who contributed to increase the renown of the politician; the intellectual, always keen on criticizing the politician, the councellor, and last but not least the ally during electoral campaigns. Both made the most of this close relationship. Brandt was helped by the talented writer who came up with numerous key phrases in his great speeches. Together, the two men invented a new political language that was sometimes successful. Grass also benefited from this extraordinary relationship. However, from 1973, as his role as an intellectual critic took a more important place, the partnership went through a critical phase. Grass, an author of extremely vitulent articles, was named by Brandt "Mr. know-it-all». Undoubtedly, his criticism let to Brandt's resignation. In spite of this outcome, the extraordinary partnership between these two characters participated in the intellectual process which lead the Federal Republic of Germany to become a Western democracy in its own eyes.
\end{abstract}

